\title{
Computational design of anti-CRISPR proteins with improved inhibition potency and expanded specificity
}

Jan Mathony $^{1 \dagger}$, Zander Harteveld ${ }^{2 \dagger}$, Carolin Schmelas ${ }^{3,4 \dagger}$, Julius Upmeier zu Belzen ${ }^{1,5,6}$, Sabine Aschenbrenner ${ }^{5,7}$, Mareike D. Hoffmann ${ }^{1,7}$, Christina Stengl ${ }^{1}$, Andreas Scheck ${ }^{2}$, Stéphane Rosset $^{2}$, Dirk Grimm ${ }^{3,4,8}$, Roland Eils ${ }^{5,6}$, Bruno E. Correia ${ }^{2 *}$ and Dominik Niopek ${ }^{1 *}$

\footnotetext{
${ }^{1}$ Synthetic Biology Group, Institute for Pharmacy and Molecular Biotechnology (IPMB) and Center for Quantitative Analysis of Molecular and Cellular Biosystems (BioQuant), University of Heidelberg, Heidelberg, 69120, Germany

${ }^{2}$ Institute of Bioengineering, École Polytechnique Fédérale de Lausanne, Lausanne, CH-1015, Switzerland and Swiss Institute of Bioinformatics (SIB), Lausanne, CH-1015, Switzerland

${ }^{3}$ Department of Infectious Diseases, Virology, University Hospital Heidelberg, Heidelberg, 69120, Germany

${ }^{4}$ BioQuant Center and Cluster of Excellence CellNetworks at Heidelberg University, Heidelberg, 69120, Germany

${ }^{5}$ Digital Health Center, Berlin Institute of Health (BIH) and Charité, Berlin, 10178, Germany

${ }^{6}$ Health Data Science Unit, University Hospital Heidelberg, Heidelberg, 69120, Germany

${ }^{7}$ Department of Theoretical Bioinformatics, German Cancer Research Center (DKFZ), Heidelberg, 69120, Germany

${ }^{8}$ German Center for Infection Research (DZIF) and German Center for Cardiovascular Research (DZHK), partner site

Heidelberg, Heidelberg, 69120, Germany

${ }^{\dagger}$ These authors contributed equally to this work.
}

*To whom correspondence should be addressed

Bruno E. Correia

bruno.correia@epfl.ch

EPFL IBI STI LPDI Station 19

CH-1015 Lausanne, Switzerland

Phone: +41 (0) 216936166
Dominik Niopek

dominik.niopek@bioquant.uni-heidelberg.de

Im Neuenheimer Feld 267

69120 Heidelberg, Germany

Phone: +49 (0) 62215451287 


\begin{abstract}
Anti-CRISPR (Acr) proteins are bacteriophage-derived antagonists of CRISPR-Cas systems. To date, Acrs were obtained either by mining sequence databanks or experimentally screening phage collections, both of which yield a limited repertoire of naturally occurring variants. Here, we applied structure-based engineering on AcrIIC1, a broad-spectrum inhibitor of type II-C CRISPR systems, to improve its efficacy and expand its specificity. We first show that fusing exogenous protein domains into AcrIIC1 dramatically enhances inhibition of the natural Neisseria meningitidis Cas9 target. Then, using structure-guided design, we converted AcrIIC1 into AcrX, a potent inhibitor of the type II-A CRISPR-Cas9 from Staphylococcus aureus widely applied for in vivo genome editing. Our work introduces designer Acrs as important biotechnological tools and provides an innovative strategy to safeguard the CRISPR technology.
\end{abstract}

The detailed characterization of bacterial CRISPR-Cas systems ${ }^{1}$ and their adaptation for precise genome engineering in mammalian cells ${ }^{2,3}$ has revolutionized the life sciences and enabled novel applications in biotechnology and medicine. The recent discovery of phage-derived anti-CRISPR proteins $^{4-6}$, i.e. potent inhibitors of Cas effectors, provides a shut-off mechanism that can keep this powerful technology in $\operatorname{check}^{7}$ and enhance the precision at which genome perturbations can be made ${ }^{8-11}$. While mining of sequence databases and screening of phage libraries proved to be powerful strategies to discover Acrs targeting a variety of Cas effectors ${ }^{5,6,12-18}$, these approaches are inherently limited to the naturally occurring protein sequence space. Moreover, for various Cas effectors of major biotechnological interest, nature might be lacking (efficient) anti-CRISPR counterparts. 
In this work, we sought to apply protein engineering to design artificial Acrs that exhibit enhanced inhibition potency and expanded target specificity (Fig. 1). As starting point, we used AcrIIC $1^{14}$, a broad-spectrum inhibitor targeting various type II-C Cas9s, including those from Neisseria meningitidis (Nme), Geobacillus stearothermophilus and Campylobacter jejuni. AcrIIC1 binds the conserved catalytic HNH domain and locks Cas9 in a DNA bindingcompetent, but catalytically inactive state. This unique inhibitory mechanism might explain why AcrIIC1 is a rather weak inhibitor ${ }^{15}$ as compared to its related proteins AcrIIC3, -C4 and -C5, all of which interfere with Cas9 DNA binding. Importantly, biochemical assays suggest tight binding of AcrIIC1 to the NmeCas9 $\mathrm{HNH}$ domain $\left(\mathrm{K}_{\mathrm{D}} \sim 6.3 \mathrm{nM}\right.$ (Ref. 2)), indicating that it is the inhibitory mechanism rather than the affinity which leads to the suboptimal performance of AcrIIC1.

We hypothesized that inserting a sufficiently large, exogenous domain into AcrIIC1 could result in an inhibitor that would still bind tightly to the HNH domain, but - on top of inhibiting Cas9 catalytic activity - could block additional, important mechanistic steps such as DNA binding (Fig. 1, left). To test this hypothesis, we first created a structural model of the AcrIIC1-Cas9sgRNA complex (see Methods for details) and interrogated it for AcrIIC1 surface sites that could be amenable for engineering. AcrIIC1 loop 5 appeared to be an ideal candidate (Supplementary Fig. 1), as it is located close to the DNA binding groove when assuming the HNH-docked Cas9 state $^{19}$, but distal from the HNH-interacting surface necessary for activity that we wished to preserve. We then created 11 different AcrIIC1 domain fusions which carry either an mCherry ( $\sim 27 \mathrm{kDa})$, Avena sativa LOV2 $(\sim 17 \mathrm{kDa})$ or PDZ domain $(\sim 9 \mathrm{kDa})$ at different positions in loop 5 and optional, flanking GS-linkers or short deletions (Supplementary Fig. 2a). These chimeric 
Acrs were screened by $\mathrm{T} 7$ endonuclease assay for their ability to inhibit NmeCas 9 cleavage of the IL2RG locus in HEK 293 T cells (Supplementary Fig. 2b). Remarkably, several AcrIIC1LOV2 and AcrIIC1-mCherry chimeras mediated highly potent NmeCas9 inhibition largely exceeding that of the parent AcrIIC1 (Fig. 2a,b, Supplementary Figs. 2b and 3). The AcrIIC1mCherry chimera \#10 (GSG-mCherry-GSG inserted between AcrIIC1 residues Y70 and A71; Supplementary Fig. 2a) showed an improvement superior to 12-fold in NmeCas9 inhibition as compared to wild-type AcrIIC1 on the four tested loci (Fig. 2a and b), and performed equivalently or better than AcrIIC3, so-far the most potent NmeCas9 inhibitor in mammalian $\operatorname{cells}^{14}$ (Fig. 2b).

To further characterize the gain in inhibition, we employed a reporter assay in which NmeCas9 cleaves a firefly luciferase transgene, thereby resulting in luciferase knockout. We co-transfected cells with the reporter, NmeCas9 and either (i) the parent AcrIIC1, (ii) the AcrIIC3 benchmark or (iii) different, engineered AcrIIC1-mCherry chimeras. During transfection, we varied the Acr:Cas9 vector ratios from 3:1 to 1:20. The chimeric inhibitors outperformed both, wild-type AcrIIC1 as well as AcrIIC3 and showed potent Cas9 inhibition even at very low Acr:Cas9 vector ratios (Fig. 2c).

To elucidate this particularly efficient inhibitory mechanism, we performed an assay in which the firefly luciferase gene was targeted by a NmeCas9 nickase carrying an impaired RuvC, but a wild-type HNH domain (see Supplementary Note for details). Luciferase activity was potently rescued in the presence of the AcrIIC1-mCherry chimeric inhibitor, comparable to the AcrIIC3 benchmark (Supplementary Fig. 4). Notably, AcrIIC1 also mediated some rescue of luciferase activity, reaching precisely the level observed when targeting the same reporter with a catalytically dead $(d) N m e$ Cas9 which inhibits firefly luciferase expression exclusively via 
CRISPR interference (Supplementary Fig. 4). This suggests that on top of blocking the catalytic activity (akin to native AcrIIC1), the chimeric inhibitors also interfere - at least to some extent with NmeCas9 DNA binding (see Supplementary Note). Together, these experiments demonstrate that domain insertion can yield anti-CRISPR proteins with far superior inhibition potency than that of natural anti-CRISPRs, thereby enabling extremely tight control of Cas9.

Having shown that AcrIIC1 could be modified to enhance its potency, we next aimed to expand its specificity. The Cas9 from Staphylococcus aureus is a type II-A CRISPR effector widely employed for in vivo genome editing ${ }^{20}$. Due to its favorable, small size $(3.2 \mathrm{~kb})$, Staphylococcus aureus (Sau)Cas9 can easily be packaged into Adeno-associated virus (AAV) particles ${ }^{20}$, which are prime vector candidates for therapeutic CRISPR applications ${ }^{21,22}$. Importantly, no antiCRISPR proteins have yet been discovered that would allow blocking SauCas9 activity. We speculated that AcrIIC1 might represent an ideal starting point to engineer an artificial SauCas9 inhibitor, as the overall fold of the SauCas9 HNH domain is similar to that of $N m e$ Cas $9^{19}$, albeit substantial differences exist at the sequence level (sequence identity is only $33.7 \%$, Supplementary Fig. 5). Recent in vitro data suggest that wild-type AcrIIC1 fails to inhibit SauCas9 function ${ }^{23}$. To independently confirm this finding, we co-expressed AcrIIC1 in HEK 293T cells together with SauCas9 and sgRNAs targeting different loci and performed T7 endonuclease assays to detect formation of insertions and deletions (indels). Strikingly, we observed a reproducible, albeit rather mild reduction of indels in the AcrIIC1 samples to about $50 \%$ of the positive controls (Supplementary Fig. 6), indicating that AcrIIC1 can also bind the SauCas9 HNH domain, though likely with a compromised affinity. 
Based on this assumption we sought to enhance SauCas9 binding. We generated a structural model of SauCas9 HNH domain in complex with AcrIIC1 and investigated the differences in the AcrIIC1 interacting surface as compared to the NmeCas9 HNH (Fig. 3). Two regions in SauCas9 HNH domain showed suboptimal contacts to corresponding AcrIIC1 residues (Fig. 3). We then performed per-residue in silico mutagenesis using Rosetta design ${ }^{24}$ followed by manual inspection, which suggested ten AcrIIC1 candidate mutations to improve binding to the SauCas9 HNH domain (Supplementary Figs. 7 and 8). We tested these mutants, first individually, in genome editing experiments targeting the EMX1 locus and then iteratively combined the most promising variants in subsequent screening rounds (Supplementary Fig. 9). Of note, we decreased the Acr:Cas9 ratio with each screening round to better resolve the performance of improving candidates. After only three rounds, we arrived at a triple mutant (N3F, D15Q, A48I; Fig. 3 and Supplementary Fig. 10), which we confirmed to be well folded in solution (Supplementary Fig. 11) and which achieved a near-complete blocking of EMX1 editing by SauCas9 (Supplementary Fig. 9). Remarkably, this mutant also retained NmeCas9 inhibitory activity comparable to wild-type AcrIIC1 (Supplementary Fig. 12, NmeCas9 samples), indicating that our engineering approach had not just re-targeted AcrIIC1 to SauCas9, but rather expanded its specificity towards this orthologue. We therefore named the mutant AcrX for its ability to target both, type II-C and II-A CRISPR effectors, which - to our best knowledge - has not yet been observed for any natural Acr.

Next, we characterized AcrX performance in detail by targeting SauCas9 to different loci (Fig. 4a). AcrX efficiently suppressed SauCas9 genome editing at all tested loci, showing up to a 30 fold improvement in inhibition as compared to the parental AcrIIC1 (Fig. 4b, Supplementary 
Figs. 13 and 14). Interestingly, akin to wild-type AcrIIC1, mCherry domain fusion to AcrX loop 5 strongly improved inhibition on NmeCas9 (Supplementary Fig. 12), but had no noticeable effect on SauCas9 inhibition. This indicates that the potent inhibition mediated by our chimeric Acrs arises from specific features of the NmeCas9 structural architecture.

Finally, to test the performance of AcrX in different mammalian cell lines and upon viral delivery, we packaged (i) AcrX as well as (ii) SauCas9 and sgRNAs targeting the EMX1, Grin2B or CXCR4 locus into AAV serotype 2. We then transduced HEK 293T (human embryonic kidney), U2OS (human osteosarcoma) or U87 (human primary glioblastoma) cells with these vectors and found that indel formation was reduced to undetectable levels in practically all samples that received AcrX (Fig. 4c).

Here, we reported the engineering of anti-CRISPR proteins with improved inhibition potency and expanded target specificity. We showed that domain insertion into AcrIIC1 enhances NmeCas9 inhibition, likely by interfering with DNA binding. On top, using structure-based design, we created the first anti-CRISPR protein able to efficiently block SauCas9 function. Our findings show that structure-guided protein engineering can yield anti-CRISPR proteins with desired properties that go beyond the currently known natural repertoire. These designer antiCRISPRs might not only find wide application in the context of biotechnology and CRISPRbased therapies ${ }^{7-10}$, but also provide an innovative strategy to safeguard CRISPR technology. 


\section{References}

1. Jinek, M. et al. Science 337, 816-821 (2012).

2. Mali, P. et al. Nat Biotechnol 31, 833-838 (2013).

3. Cong, L. et al. Science 339, 819-823 (2013).

4. Bondy-Denomy, J., Pawluk, A., Maxwell, K.L. \& Davidson, A.R. Nature 493, 429-432 (2013).

5. Bondy-Denomy, J. et al. Nature 526, 136-139 (2015).

6. Pawluk, A., Bondy-Denomy, J., Cheung, V.H., Maxwell, K.L. \& Davidson, A.R. MBio 5, e00896 (2014).

7. Basgall, E.M. et al. Microbiology 164, 464-474 (2018).

8. $\quad$ Bubeck, F. et al. Nat Methods 15, 924-927 (2018).

9. Hoffmann, M.D. et al. Nucleic Acids Res gkz271 (2019).

10. Nakamura, M. et al. Nat Commun 10, 194 (2019).

11. Shin, J. et al. Sci Adv 3, e1701620 (2017).

12. Rauch, B.J. et al. Cell 168, 150-158.e110 (2016).

13. Pawluk, A. et al. Nat Microbiol 1, 16085 (2016).

14. Pawluk, A. et al. Cell 167, 1829-1838 e1829 (2016).

15. Lee, J. et al. MBio 9, e02321-02318 (2018).

16. Hynes, A.P. et al. Nat Commun 9, 2919 (2018).

17. Watters, K.E., Fellmann, C., Bai, H.B., Ren, S.M. \& Doudna, J.A. Science 362, 236-239 (2018).

18. Marino, N.D. et al. Science 362, 240-242 (2018).

19. Harrington, L.B. et al. Cell 170, 1224-1233.e1215 (2017).

20. Ran, F.A. et al. Nature 520, 186-191 (2015).

21. Senís, E. et al. Biotechnology Journal 9, 1402-1412 (2014).

22. Schmidt, F. \& Grimm, D. Biotechnol J 10, 258-272 (2015). 
23. Seamon, K.J., Light, Y.K., Saada, E.A., Schoeniger, J.S. \& Harmon, B. Anal Chem 90, 6913-6921 (2018).

24. Leaver-Fay, A. et al. Methods Enzymol 487, 545-574 (2011).

Acknowledgments: We thank the Synthetic Biology (IPMB, Heidelberg University), Virus-host interactions (Heidelberg University clinics) and the Protein Design and Immunoengineering groups (EPFL, Lausanne) for helpful discussions, Kathleen Börner (Heidelberg University clinics, Heidelberg, Germany) and Karsten Rippe (DKFZ, Heidelberg, Germany) for providing cell lines and Katharina Niopek for critical reading of the manuscript. We would like to thank the EPFL's Scientific IT and Application Support Center for their support on computational infrastructure. We would like to thank the Protein Production and Structure Core facility for their support on the protein biophysical characterization experiments. This study was funded by the Helmholtz association, the German Research Foundation (DFG) and the Federal Ministry of Education and Research (BMBF) (R.E.). D.G. is grateful for funding from the German Center for Infection Research (DZIF, TTU-HIV 04.803 and TTU-HIV 04.815) and the Cystic Fibrosis Foundation Therapeutics (CFFT, grant number GRIMM15XX0). C.S., D.G. and R.E. acknowledge funding from the Transregional Collaborative Research Center TRR179 (DFG, Projektnummer 272983813). C.S. and D.G. acknowledge additional funding by the Cluster of Excellence CellNetworks (DFG, EXC81). B.E.C. is a grantee from the European Research Council (Starting grant - 716058), the Swiss National Science Foundation and the Biltema Foundation. The computational simulations were performed at the CSCS - Swiss National Supercomputing Centre through a grant obtained by B.E.C. Z.H. is supported by a grant from the Swiss National Science Foundation. S.R. is supported by a grant of the National Center of Competence in Research in Chemical Biology. 
Author contributions: D.N. conceived the initial idea and refined it together with J.U.z.B. and B.E.C.; J.M, C.S., S.A. and D.N. designed and performed experiments. S.A. and M.D.H. performed AAV production. S.R. purified the Acrs and performed protein-biochemical characterization. Z.H., J.U.z.B., A.S. and B.E.C. performed in silico structural analysis and modeling. D.G. provided expertise on AAVs and SauCas9. B.E.C. and D.N. jointly directed the work. B.E.C. and D.N. wrote the manuscript with support from all authors.

Competing interests: None of the authors have competing financial or non-financial interests to declare. 


\section{Figures and legends}

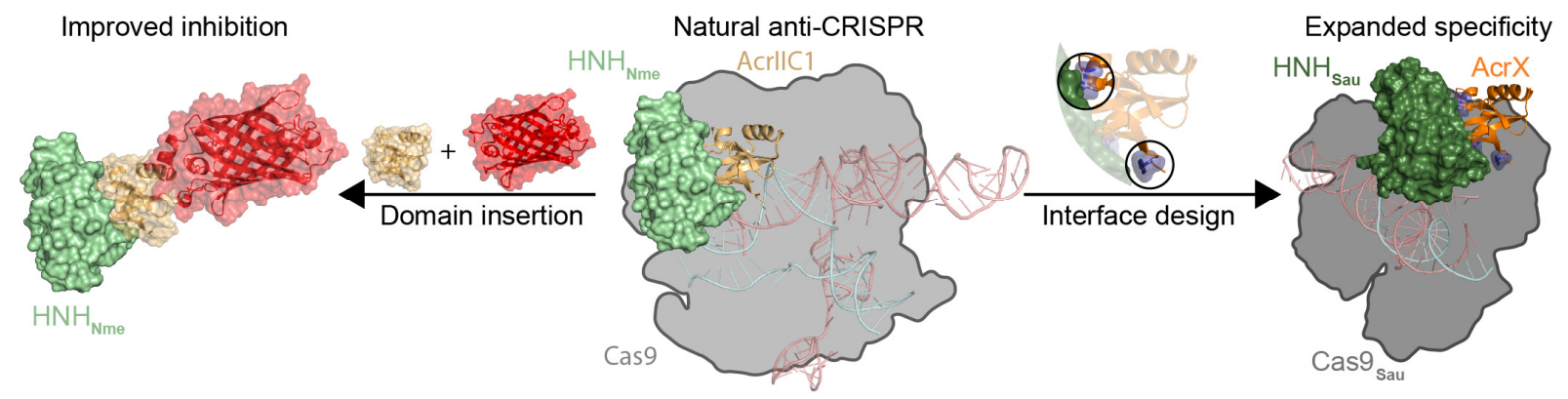

Fig. 1 | Improving potency and expanding specificity of anti-CRISPR proteins. Domain

insertion into AcrIIC1 loop 5 yields chimeric inhibitors with improved inhibition potency, while structure-based engineering of its HNH-binding interface expands its target specificity (PDB 4ZIN, 5VGB, 5F9R and 5CZZ). 

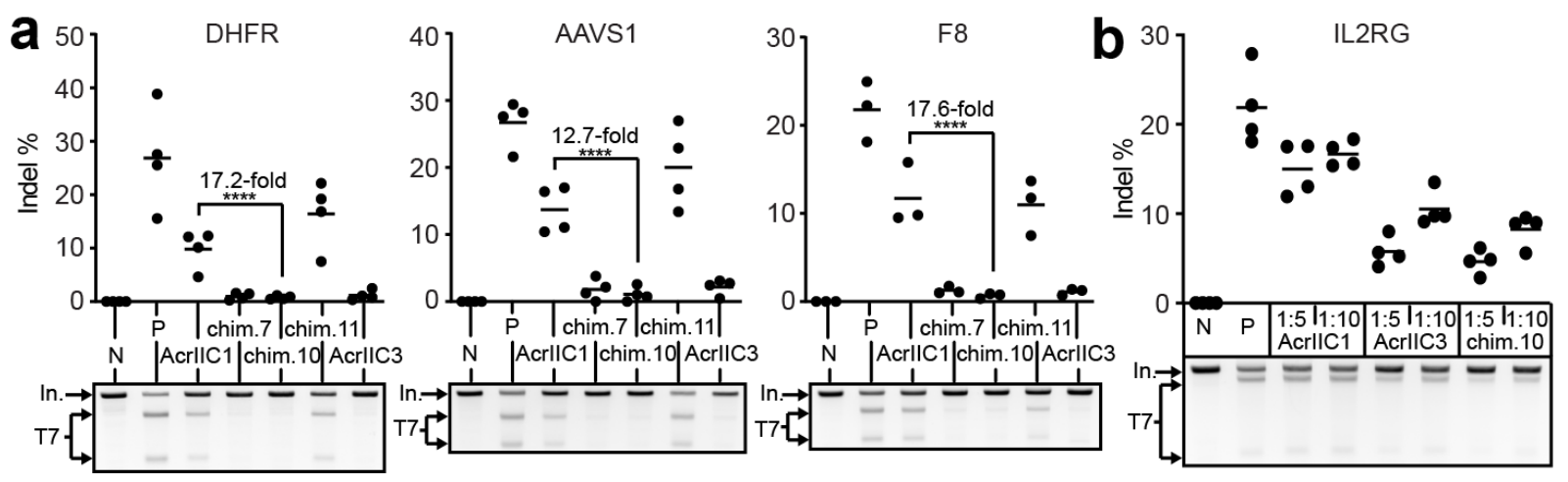

C

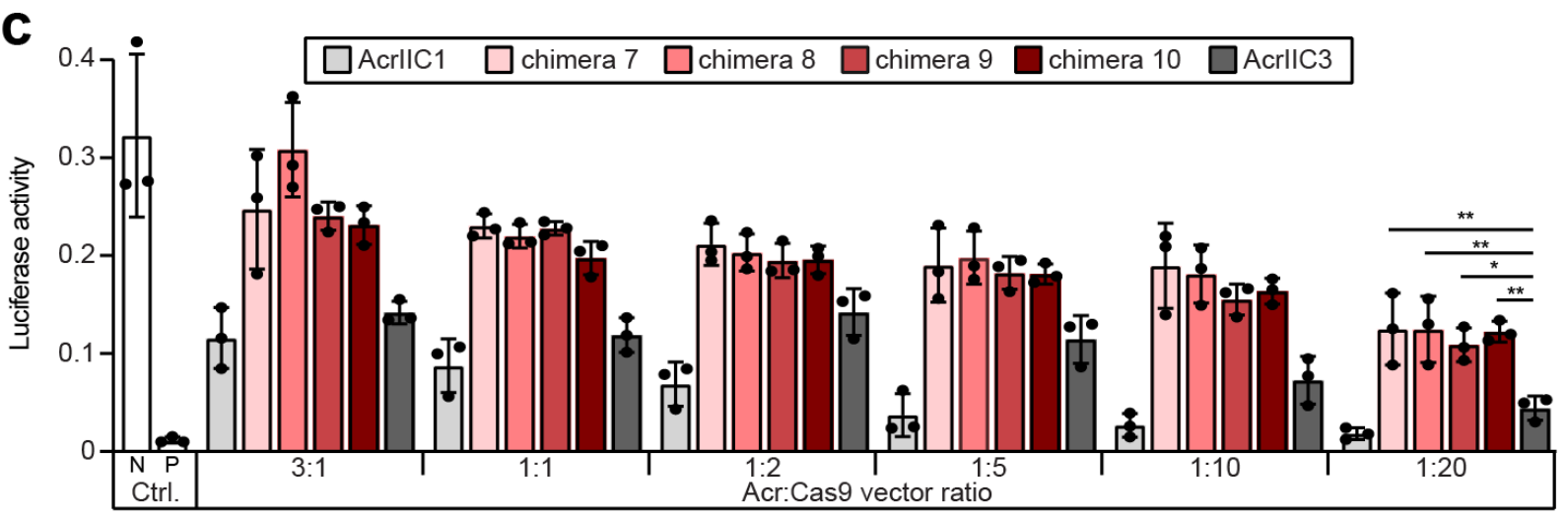

Fig. 2 | Domain insertion into AcrIIC1 yields a highly potent NmeCas9 inhibitor.

(a,b) HEK 293T cells were co-transfected with vectors expressing NmeCas9, the indicated Acr and sgRNAs targeting different genomic loci followed by $\mathrm{T} 7$ endonuclease assay. In a, Acr:NmeCas9 vector ratio used during transfection was 1:1, while in $\mathbf{b}$, the indicated, low Acr:NmeCas9 vector ratios were used. Representative $\mathrm{T} 7$ gel images and corresponding quantification of indel frequencies are shown. Lines in plots show means, dots are individual data points for $\mathrm{n}=4$ (DHFR, AAVS1 and IL2RG locus) or $\mathrm{n}=3$ (F8 locus) independent experiments. Chim., AcrIIC1-mCherry chimeras in Supplementary Fig. 2. In., input band. T7, T7 cleavage fragments. (c) AcrIIC1-mCherry chimeras outperform wild-type AcrIIC1 and AcrIIC3. Cells were co-transfected with vectors encoding NmeCas9, a firefly luciferase reporter and corresponding reporter-targeting sgRNA as well as the indicated Acr followed by luciferase assay. Bars indicate means, error bars the SD for $\mathrm{n}=3$ independent experiments. (a-c) $\mathrm{N}$, negative (Cas 9 only (a,b) or reporter only (c) control (Ctrl.)). P, positive control (Cas9 + sgRNA 
bioRxiv preprint doi: https://doi.org/10.1101/685032; this version posted June 27, 2019. The copyright holder for this preprint (which was not certified by peer review) is the author/funder, who has granted bioRxiv a license to display the preprint in perpetuity. It is made available under aCC-BY-NC 4.0 International license.

$(\mathbf{a}, \mathbf{b})$ or reporter + Cas9 $(\mathbf{c})) \cdot{ }^{*} P<0.05, * * P<0.01, * * * P<0.001, * * * * P<0.0001$ by one-way ANOVA with Bonferroni correction. 

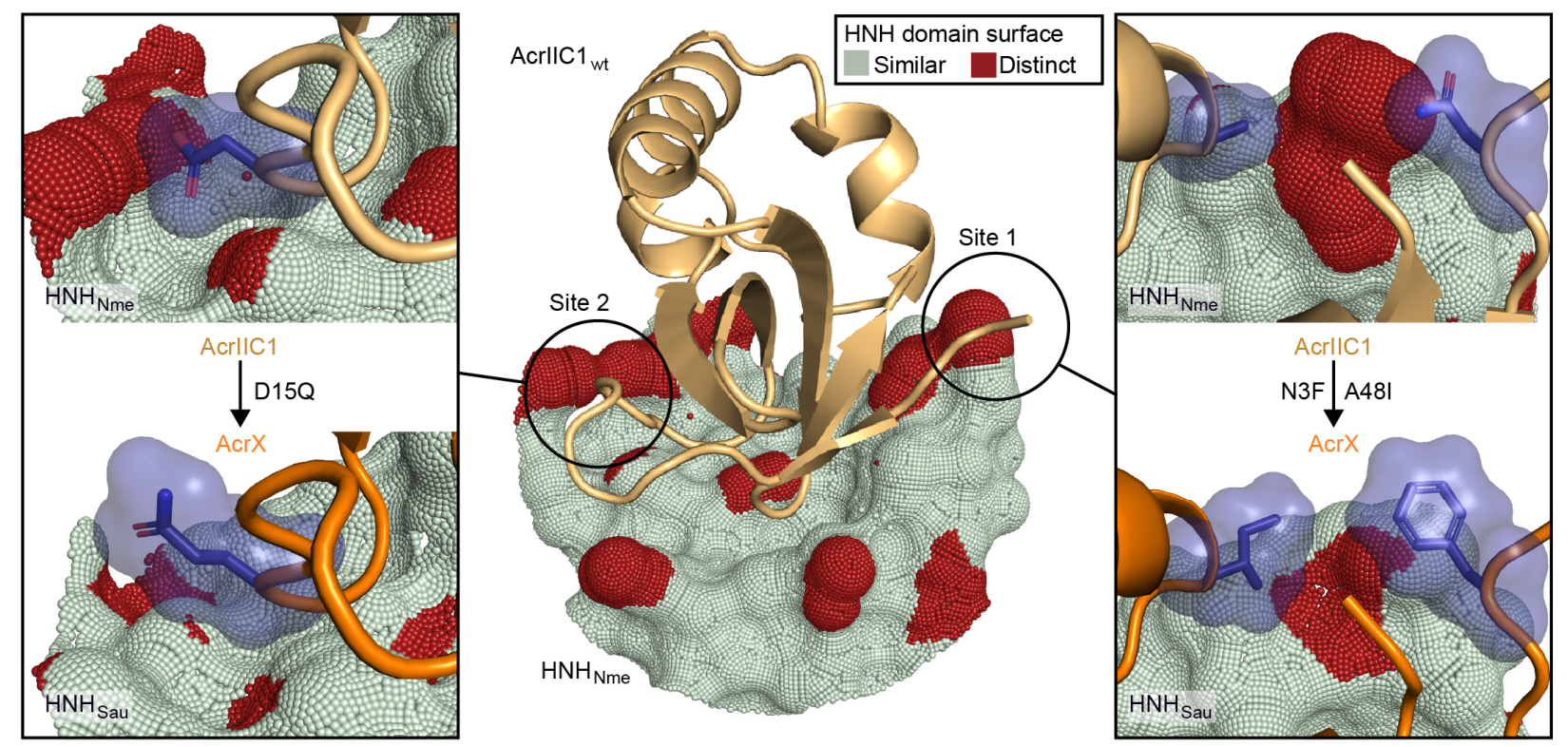

Fig. 3 | Structure-guided design of AcrX, an anti-CRISPR protein targeting S. aureus Cas9.

Center: Structure showing AcrIIC1 binding to the NmeCas9 HNH domain surface. Red patches indicate regions at which the SauCas9 $\mathrm{HNH}$ surface displays deviations of at least $1 \AA$ as compared to the NmeCas9 HNH surface, highlighting the most important sites to target by mutagenesis. Left/right: Comparison of wild-type AcrIIC1 residues (D15 (left), N3 and A48 (right)) binding to the NmeCas9 HNH surface to the corresponding, engineered residues (15Q (left), 3F and 48I (right)) binding to the SauCas9 HNH surface. 
a

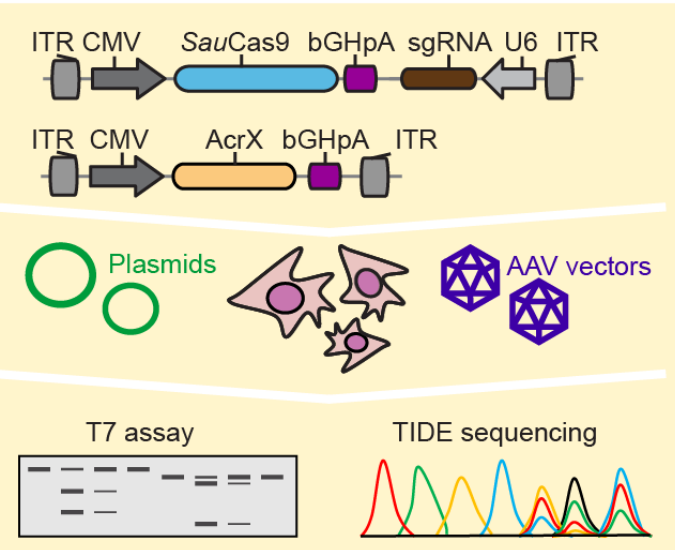

CCR5 Acr:Cas9 = 2:1

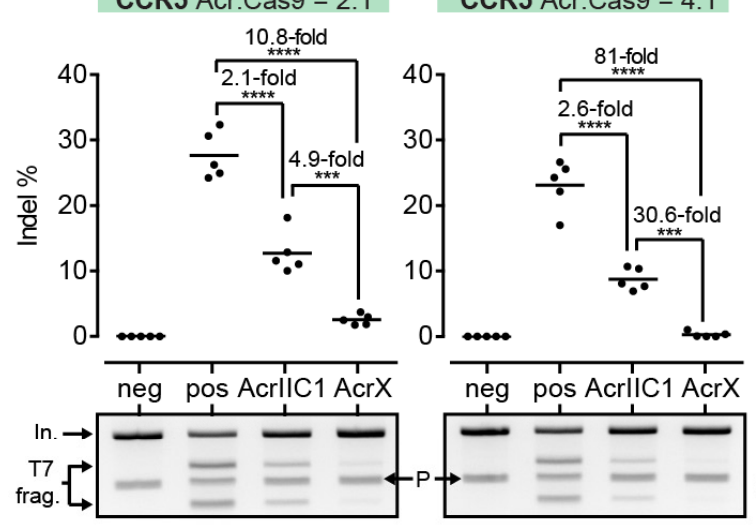

b

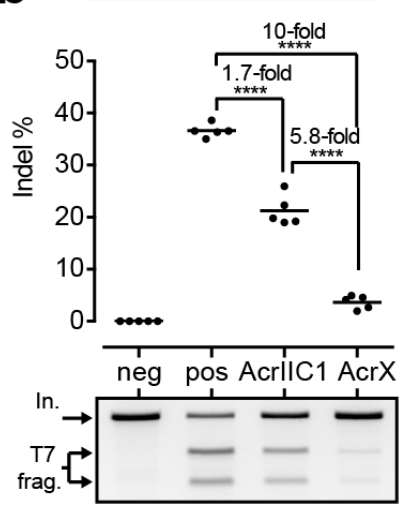

HBB Acr:Cas9 = 4:1

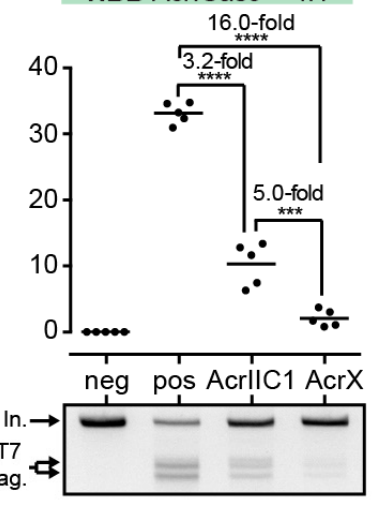

U2OS (bone osteosarcoma)

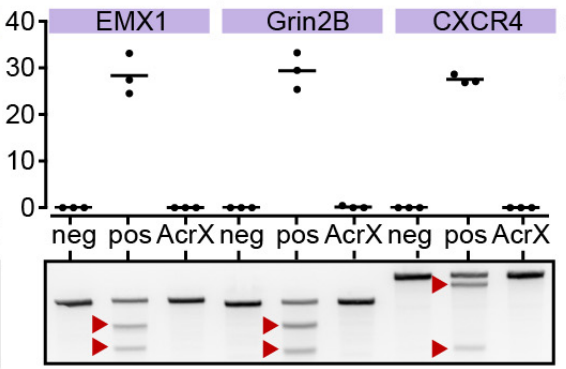

EMX1 Acr:Cas9 = 2:1

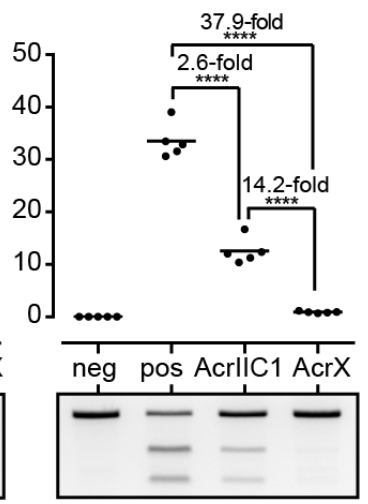

Grin2B Acr:Cas9 $=4: 1$

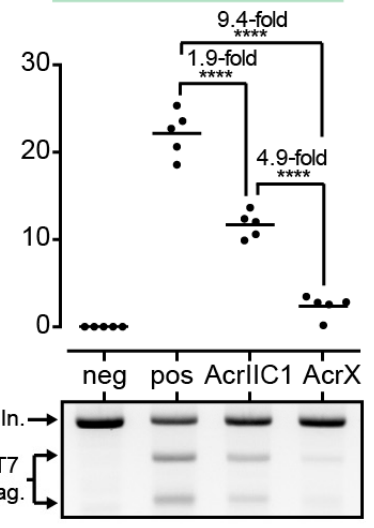

U87 (primary glioblastoma)

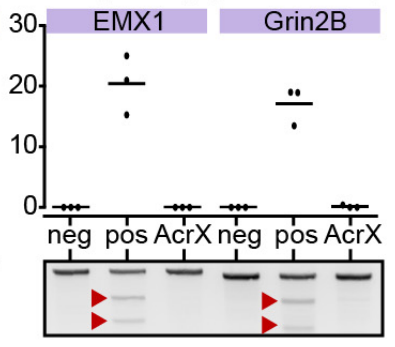

Fig. 4 | Characterization of AcrX, an engineered S. aureus Cas9 inhibitor. (a) Schematics of vectors and experimental setup. (b) HEK 293T cells were co-transfected with the vectors in a targeting the indicated loci followed by $\mathrm{T} 7$ endonuclease assay. The Acr:Cas9 vector ratio used during transfection is indicated. P denotes a T7 cleavage band which is due to a polymorphism in the CCR5 gene (Supplementary Fig. 15). In., input band. T7 frag., T7 cleavage fragments. $* * * P$ $<0.001,{ }^{* * * *} P<0.0001$ by one-way ANOVA with Bonferroni correction. (c) AAV-mediated 
delivery of AcrX results in potent SauCas9 inhibition in different cell lines. Cells were cotransduced with AAV2 vectors expressing (i) SauCas9 and a sgRNA targeting the indicated loci and (ii) AcrX followed by T7 endonuclease assay. Red triangles point to T7 cleavage fragments. (b,c) Representative T7 gel images and corresponding quantification of indel frequencies are shown. Lines in the plots indicate means, dots individual data points for $n=5$ (b) or $n=3$ (c) independent experiments. Neg, negative control (Cas9 only). Pos, positive control (Cas9 + sgRNA). 


\section{Online Methods}

\section{Modeling of AcrIIC1-mCherry fusions}

We used the Rosetta remodel application ${ }^{25}$ to generate the AcrIIC1-mCherry chimeras based on the structures for AcrIIC1 (PDB 5VGB) and mCherry (PDB 4ZIN). The N- and C-termini of mCherry were absent in the crystal structure and were rebuilt using fragment insertion together with cyclic coordinate descent ${ }^{26}$ and kinematic closure ${ }^{27,28}$ with default values. For the designed chimera with a two-residue deletion, approximately 1500 decoys were generated and subsequently clustered with a root mean square deviation (r.m.s.d) threshold of $5 \AA$ into 27 clusters. For the chimera with additional GSG-linkers at the N- and C-termini, approximately 1200 structures were clustered in 100 clusters with the same parameters. Representative examples of the three most populated clusters, which also have the lowest energies, are shown to illustrate the potential structural diversity of the AcrIIC1-mCherry chimeras (Supplementary Fig. 3). Analysis of the Rosetta outputs, structural models and the biochemical data was performed using the rstoolbox ${ }^{29}$.

\section{AcrIIC1 interface design}

To screen in silico for mutations that could enhance the affinity of AcrIIC1 to Staphylococcus aureus (Sau)Cas9, we modeled a complex of AcrIIC1 (originally crystalized with the HNH domain of Neisseria meningitidis (Nme)Cas9, PDB 5VGB) with the SauCas9 HNH domain (PDB 5CZZ). A first structural alignment was performed between the Nme and SauCas9 domains using TM-align ${ }^{30}$ revealing an r.m.s.d of $2.32 \AA$ and several structurally and sequenceconserved interface regions. These conserved interface regions were then used to refine the alignment, using the PyMOL (version 2.3.1) superposition function, to obtain the modeled 
complex used for the design simulations (Supplementary Fig. 5). We then analyzed the interfaces of both orthologues to pinpoint hotspots which could be designed in AcrIIC1 to enhance its interaction with SauCas9. These hotspots were visualized with surface point-wise distances between the two surfaces that were computed with a custom script. For each point on the reference surface (Nme), the distance to the closest point on the other surface (Sau) was calculated. In the visualizations, these distances were binarized by setting a cutoff of $1 \AA$ (Fig. 3).

Next, we used RosettaScripts ${ }^{31}$ to perform a single-site in silico mutagenesis, thereby allowing subsets of amino acids for each of the selected residues on AcrIIC1. From our interface analysis we selected the following residues in AcrIIC1 for in silico mutagenesis: N3, D15, R36, D43, D45, D46, K47, A48 and M77. The design protocol consisted of two rounds of packing and minimization with fixed backbone. We generated a total of 51 designs and computed their change in binding free energy (ddG), number of hydrogen bonds across the interface, change in hydrophobic solvent-accessible surface area (SASA), and interface shape complementary with the target SauCas9 HNH domain. Designs with improved ddGs compared to that of the AcrIIC1SauCas9 complex (-22 Rosetta energy units), increased hydrogen bonds across the interface, improvements in SASA and shape complementarity were manually inspected. A total of 10 substitutions on 8 sites were selected for experimental validation. Mutations N3F, N3Y and A48I were designed to increase interface packing and pi-stacking with the complementary hydrophobic patches on SauCas9. D43F and D45F were generated to fill voids within the interface boundaries and increase the hydrophobic packing. D15Q, R36D, D46E, K47Q and M77S were introduced to balance the underlying charge distribution on SauCas9 within the respective region. The overall design workflow is shown in Supplementary Fig. 7. 
After experimental validation and combination of the proposed mutations, the final AcrX (AcrIIC1 with N3F, D15Q, A48I) was modeled following the same protocol. Electrostatic properties for AcrIIC1, AcrX as well as the NmeCas9 and the SauCas9 HNH domains were computed using the adaptive Poisson Boltzmann solver (APBS) plugin in PyMOL

(Supplementary Fig. 10). The mutation D15Q results in a less negative potential, to optimize the interaction with a patch of the interface in SauCas9 that has a lower positive potential as compared to NmeCas9.

\section{Protein expression and purification}

DNA sequences of the designs were purchased from Twist Bioscience. For bacterial expression,

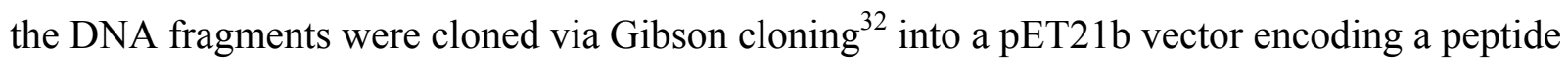
sequence containing a tobacco etch virus (TEV) protease cleavage site followed by a terminal His-tag and transformed into E. coli BL21(DE3). Expression was conducted in Terrific Broth supplemented with ampicillin $(100 \mu \mathrm{g}$ per ml). Cultures were inoculated at an OD600 of 0.1 from an overnight culture and incubated in a shaker at $37^{\circ} \mathrm{C}$ and $220 \mathrm{rpm}$. After reaching an OD600 of 0.6 , expression was induced by the addition of $0.4 \mathrm{mM} \mathrm{IPTG}$ and cells were further incubated overnight at $20^{\circ} \mathrm{C}$. Cells were harvested by centrifugation and pellets were resuspended in lysis buffer (50 mM TRIS, pH 7.5, $500 \mathrm{mM} \mathrm{NaCl}, 5 \%$ glycerol, $1 \mathrm{mg}$ per ml lysozyme, $1 \mathrm{mM}$ PMSF, $4 \mu \mathrm{g}$ per ml DNase). Resuspended cells were sonicated and clarified by centrifugation. Ni-NTA purification of sterile-filtered $(0.22 \mu \mathrm{m})$ supernatant was performed using a $5 \mathrm{ml}$ His-Trap FF column on an ÄKTA pure system (GE Healthcare). Bound proteins were eluted using an imidazole concentration of $300 \mathrm{mM}$. Concentrated proteins were further 
purified by size exclusion chromatography on a Hiload 16/600 Superdex 75 pg column (GE

Healthcare) using PBS buffer (pH 7.4) as mobile phase.

\section{Circular dichroism (CD)}

Far-UV circular dichroism spectra of AcrIIC1 and AcrX were collected between a wavelength of $190 \mathrm{~nm}$ to $250 \mathrm{~nm}$ on a Jasco J-815 CD spectrometer in a $1 \mathrm{~mm}$ path-length quartz cuvette.

Proteins were dissolved in $10 \mathrm{mM}$ phosphate buffer at concentrations between $20 \mu \mathrm{M}$ and $40 \mu \mathrm{M}$. Wavelength spectra were averaged from two scans with a scanning speed of $20 \mathrm{~nm}$ per min and a response time of $0.125 \mathrm{sec}$. The thermal denaturation curves were collected by measuring the change in ellipticity at $220 \mathrm{~nm}$ from 20 to $90^{\circ} \mathrm{C}$ with 2 or $5^{\circ} \mathrm{C}$ increments.

Size-exclusion chromatography combined with Multi-Angle Light-Scattering (SEC-MALS)

Multi-angle light scattering was used to assess the monodispersity and molecular weight of the proteins. Samples containing 50-100 $\mu \mathrm{g}$ of protein in PBS buffer ( $\mathrm{pH} 7.4)$ were injected into a Superdex 75 10/300 GL column (GE Healthcare) using an HPLC system (Ultimate 3000, Thermo Scientific) at a flow rate of $0.5 \mathrm{ml}$ per min coupled in-line to a multi-angle light scattering device (miniDAWN TREOS, Wyatt). Static light-scattering signal was recorded from three different scattering angles. The scatter data were analyzed by ASTRA software (version 6.1, Wyatt).

\section{Construct design and cloning}

Constructs used in this study are listed in Supplementary Table 1. Sequences for all plasmids created in this study are provided as GenBank files in Supplementary Data. The following 
constructs were generated via classical restriction enzyme cloning or Golden Gate assembly ${ }^{33}$.

Oligonucleotides and synthetic double-stranded DNAs were obtained from IDT. PCRs were performed either with Q5 Hot Start high-fidelity DNA polymerase (New England Biolabs) or Phusion Flash high-fidelity polymerase (Thermo Fisher Scientific). After separating PCR products or restriction digest products on agarose gels, bands of the desired size were cut out and the DNA was extracted using the QIAquick gel extraction kit (Qiagen). Restriction enzymes and T4 DNA ligase were obtained from Thermo Fisher Scientific. Constructs were transformed into chemical competent Top10 cells (Thermo Fisher Scientific). DNA was purified using the QIAamp DNA Mini, Plasmid Plus Midi or Plasmid Maxi kit (all from Qiagen). The plasmid pEJS654 All-in-One AAV-sgRNA-hNmeCas9 co-encoding NmeCas9 and a corresponding sgRNA expression cassette was a kind gift from Erik Sontheimer (Addgene \#112139). The plasmid pX601-AAV-CMV::NLS-SaCas9-NLS-3xHA-bGHpA;U6::BsaI-sgRNA co-encoding SauCas9 and a corresponding sgRNA expression cassette was a kind gift from Feng Zhang (Addgene \#61591). The luciferase reporter plasmid was previously reported by us ${ }^{8}$ and modified as follows: an NTS33 target site $^{34}$ was inserted behind the firefly luciferase start codon and in frame with the luciferase gene; an NTS33-targeting sgRNA was subsequently inserted into the modified reporter. Vectors encoding AcrIIC1, AcrIIC3 and AcrIIA4 were previously reported by us ${ }^{8,9}$. AsLOV2-, PDZ- and mCherry-encoding sequences were obtained from IDT as human-codon-optimized synthetic DNA fragments (gBlocks). AcrIIC1 chimeras were created by Golden Gate cloning as follows: the plasmid encoding AcrIIC1 was first linearized at a selected position in the AcrIIC1 coding sequence via around-the-horn PCR; LOV2-, PDZ- and mCherrycoding sequences were then amplified by matching primers introducing optional GS linker encoding sequences and ligated into the linearized vector backbone; point mutations and protein 
tags were introduced via around-the-horn PCR via the primer overhangs. Annealed

oligonucleotides corresponding to the target site sequence were cloned into the hybrid Cas9sgRNA vectors via SapI (NmeCas9) or BsaI (SauCas9) restriction sites as described previously ${ }^{20,}$ 34.

\section{Cell culture and AAV lysate production}

HEK 293 T (human embryonic kidney), U87 (human primary glioblastoma; kindly provided by Kathleen Börner, Heidelberg University clinics) and U2OS (human osteosarcoma; kindly provided by Karsten Rippe, German Cancer Research Center (DKFZ), Heidelberg) were cultured at $5 \% \mathrm{CO}_{2}$ and $37^{\circ} \mathrm{C}$ in a humidified incubator and maintained in phenol red-free Dulbecco's Modified Eagle Medium (DMEM; ThermoFisher/GIBCO) supplemented with $10 \%$ (v/v) fetal calf serum (Biochrom AG), $2 \mathrm{mM} \mathrm{L-glutamine}$ and $100 \mathrm{U}$ per $\mathrm{ml}$ penicillin and $100 \mu \mathrm{g}$ per $\mathrm{ml}$ streptomycin (both ThermoFisher/GIBCO). The U2OS medium was additionally supplemented with $1 \mathrm{mM}$ sodium pyruvate (GIBCO). Cell lines were free of mycoplasma contamination and authenticated prior usage (Multiplexion, Heidelberg, Germany).

AAV-containing cell lysates were produced by seeding HEK 293T cells into 6-well plates (Corning) at a density of 350,000 cells per well. On the next day, cells were co-transfected using $8 \mu 1$ Turbofect reagent (ThermoFisher Scientific) per well and 1,333 ng of each of the following plasmids: (i) An AAV vector plasmid carrying the transgenes to be delivered flanked by inverted terminal repeats (ITRs), (ii) an AAV helper plasmid carrying rep and cap genes of AAV

serotype 2 and (iii) an adenoviral helper plasmid providing the required helper functions ${ }^{35}$. The AAV vector plasmid encoded either (1) a dual transgene cassette expressing SauCas9 driven from a CMV promoter and sgRNA driven from a shortened U6 promoter, targeting either the 
EMX1, Grin2B or CXCR4 locus, in a single-stranded AAV context, (2) the same SauCas9

cassette but together with an empty sgRNA expression cassette (negative control) or (3) a CMV promoter-driven AcrX transgene in a double-stranded AAV context. Three days after transfection, cells were harvested in $300 \mu \mathrm{l}$ PBS and lysed by subjecting them to five alternating freeze-thaw cycles in liquid nitrogen and in a $37^{\circ} \mathrm{C}$ water bath. Cell debris was separated by centrifugation and the supernatant containing the AAVs was stored at $4^{\circ} \mathrm{C}$ (for a maximum of two weeks) prior to usage.

\section{Luciferase reporter assays}

HEK 293 T cells were seeded into 96-well plates at a density of 12,500 cells per well. For titration experiments employing the chimeric Acrs (Fig. 2c), the cells were co-transfected on the following day with (i) 33 ng of a dual luciferase reporter plasmid encoding a firefly and Renilla luciferase gene and an sgRNA targeting the NTS33 site in the firefly reporter gene, (ii) 33 ng of a vector co-expressing NmeCas9 and a sgRNA targeting the NTS33 site, (iii) 99, 33, 16.5, 6.6, 3.3 or $1.65 \mathrm{ng}$ of Acr vector, and (iv) 0, 66, 82.5, 92.4, 95.7 or $97.35 \mathrm{ng}$ of an irrelevant stuffer plasmid (pBluescript), respectively. The stuffer plasmid was added to keep the total amount of DNA transfected constant in all samples. For dNmeCas9 or nNmeCas9 experiments (Supplementary Fig. 4), 33 ng of the dual luciferase reporter, 33 ng of d- or nNmeCas9 and 33 ng Acr construct were co-transfected. Transfections were performed using Lipofectamine 3000 reagent (Thermo Fisher Scientific) according to the manufacturer's protocol.

Two days post-transfection, the cells were washed with 1x PBS and lysed with $30 \mu 1$ passive lysis buffer (Promega) for $30 \mathrm{~min}$, while being shaken on a thermomixer (Eppendorf) at $500 \mathrm{rpm}$ and at room temperature. Finally, luciferase activity was analyzed using the Dual-Glo luciferase 
assay system (Promega). In short, $10 \mu \mathrm{l}$ of lysate were transferred to a white sample plate and photo counts were measured with a GLOMAX 96 microplate luminometer (Promega).

Integration time was $10 \mathrm{~s}$ with a delay of $2 \mathrm{~s}$ between substrate injection and measurement. To calculate the reported luciferase activity values, firefly luciferase photon counts were normalized those obtained for Renilla luciferase.

\section{T7 endonuclease assay and TIDE sequencing}

Genomic target sites relevant for T7 and TIDE experiments are listed in Supplementary Table 2. For transfection-based experiments, HEK $293 \mathrm{~T}$ cells were seeded in 96-well plates (Eppendorf) at a density of 12,500 cells per well. For AAV transduction-based experiments, HEK 293T, U2OS and U87 cells were seeded at a density of 3,500, 3,000 and 3,000 cells per well, respectively. For experiments with SauCas9 (Fig 4b, Supplementary Figs. 13 and 14), transfections were performed with JetPrime using $0.3 \mu 1$ of JetPrime reagent per well, except for the experiment shown in Supplementary Fig. 12, in which Lipofectamine 3000 was employed for transfection of all samples including those with SauCas9. Note that the CXCR4 target site in Supplementary Fig. 13 is the CXCR4-1 site in Supplementary Table 2. For NmeCas9 experiments (Fig. 2a,b), transfections were conducted with Lipofectamine 3000 using $0.2 \mu 1$ Lipofectamine reagent, $0.4 \mu \mathrm{l}$ p3000 and $200 \mathrm{ng}$ total DNA per well. Cells were co-transfected with 100,133 or $160 \mathrm{ng}$ of Acr vector and 100, 67 or $40 \mathrm{ng}$ or all-in-one Cas9/sgRNA vector corresponding to Acr:Cas9 vector ratios of 1:1, 2:1 and 4:1, respectively, as indicated in the figures. Transfections for the initial screen of the chimeric AcrIIC1 variants (Supplementary Fig. 2) were performed with only 100 ng of total DNA per well, using a 1:1 ratio of Cas9/sgRNA and Acr vectors. 
For AAV-based experiments (Fig. 4c), cells were co-transduced with $50 \mu 1$ of AcrX and $50 \mu 1$ Cas9/sgRNA AAV lysates on two subsequent days. As negative and positive controls, cells were transduced with $50 \mu 1$ of Cas9-only AAV lysate or Cas9/sgRNA AAV lysate (see above), respectively, topped up to $100 \mu \mathrm{l}$ with PBS (to keep the transduction volume identical in all samples). Note, the CXCR4-target site in Fig. 4c is the CXCR4-2 site in Supplementary Table 2. Three days post-transfection or (initial) transduction, cells were harvested in DirectPCR Lysis Reagent (Peqlab) supplemented with Proteinase K (Sigma), and incubated at $55^{\circ} \mathrm{C}$ for at least 6 hours followed by Proteinase $\mathrm{K}$ inactivation at $85^{\circ} \mathrm{C}$ for $45 \mathrm{~min}$. The CRISPR-Cas9-targeted genomic loci were then amplified via PCR with appropriate primers (Supplementary Table 3) using Q5 Hot Start High-Fidelity DNA Polymerase (New England Biolabs). Indel frequencies were assessed by $\mathrm{T} 7$ endonuclease assay or TIDE sequencing ${ }^{36}$.

For T7 assays, $5 \mu 1$ of the target amplicons were diluted 1:4 in 1x NEB buffer 2 and subsequently denatured at $95^{\circ} \mathrm{C}$ for 5 min and re-annealed by applying a ramp rate of $-2^{\circ} \mathrm{C}$ per sec at 95 to $85^{\circ} \mathrm{C}$ and $-0.1^{\circ} \mathrm{C}$ per sec at 85 to $25^{\circ} \mathrm{C}$ using a nexus GSX1 Mastercycler (Eppendorf).

Subsequently, $0.5 \mu 1$ of T7 endonuclease (New England Biolabs) was added, and samples were incubated at $37^{\circ} \mathrm{C}$ for $15 \mathrm{~min}$, followed by analysis on a $2 \%$ TBE agarose gel. The PCR input and T7 cleavage fragment bands were then quantified using the gel analysis tool in Image ${ }^{37,38}$ (http://imagej.nih.gov/ij/). The frequency of insertions and deletions was calculated using the formula indel $(\%)=100 \times\left(1-(1-\text { fraction cleaved })^{1 / 2}\right)$, whereas the fraction cleaved $=$ Sum(cleavage product bands)/Sum(cleavage product bands + PCR input band). Full-length gel images are shown in Supplementary Fig. 16.

For TIDE sequencing analysis, the target locus PCR amplicon was purified from a $1 \%$ agarose gel using the QIAquick Gel Extraction Kit (Qiagen). The DNA concentration was determined 
using a nano-photometer (Nanodrop, Thermo Fisher Scientific) and DNA was diluted to a final concentration of $75 \mathrm{ng}$ per $\mu \mathrm{l}$ and sent for Sanger sequencing (Eurofins, Germany). Percentages of modified sequences were then quantified using the TIDE web tool (https://tide.deskgen.com/).

\section{Statistical analysis}

Individual data points correspond to independent experiments with cells that were seeded and transfected/transduced independently and on different days. Each data point shown for the luciferase experiments further represents the mean of three technical replicates, i.e. cell cultures in different wells that were transfected and treated in parallel. Reported differences between groups were analyzed for statistical significance by one-way ANOVA and Bonferroni's corrected post-hoc test. One, two, three and four asterisks thereby indicated $P$ values below 0.05 , $0.01,0.001$ and 0.0001 , respectively. $P$ values $<0.05$ were considered statistically significant.

\section{Reporting Summary}

Further information on experimental design is available in the Life Sciences Reporting Summary linked to this article.

\section{Data and code availability}

Vectors encoding AcrX, AcrX* as well as the AcrIIC1-mCherry chimera will be made available via Addgene (plasmids \# 128112-128114). Annotated vector sequences (GenBank files) are provided in Supplementary Data. Code and data for the computational domain assembly and the design of the improved AcrIIC1 point mutants will be made available on GitHub 
(https://github.com/zanderharteveld/AcrX). Additional data can be obtained from the corresponding authors on request.

\section{References (Online Methods)}

8. $\quad$ Bubeck, F. et al. Nat Methods 15, 924-927 (2018).

9. Hoffmann, M.D. et al. Nucleic Acids Res gkz271 (2019).

20. Ran, F.A. et al. Nature 520, 186-191 (2015).

25. Huang, P.S. et al. PLoS One 6, e24109 (2011).

26. Canutescu, A.A. \& Dunbrack, R.L., Jr. Protein Sci 12, 963-972 (2003).

27. Coutsias, E.A., Seok, C., Jacobson, M.P. \& Dill, K.A. J Comput Chem 25, 510-528 (2004).

28. Mandell, D.J., Coutsias, E.A. \& Kortemme, T. Nat Methods 6, 551-552 (2009).

29. Bonet, J., Harteveld, Z., Sesterhenn, F., Scheck, A. \& Correia, B.E. BMC Bioinformatics 20, 240 (2019).

30. Zhang, Y. \& Skolnick, J. Nucleic Acids Res 33, 2302-2309 (2005).

31. Fleishman, S.J. et al. PLoS One 6, e20161 (2011).

32. Gibson, D.G. et al. Nat Methods 6, 343-345 (2009).

33. Engler, C. \& Marillonnet, S. Methods Mol Biol 1073, 141-156 (2013).

34. Amrani, N. et al. Genome Biol 19, 214 (2018).

35. Herrmann, A.K. et al. ACS Synth Biol 8, 194-206 (2019).

36. Brinkman, E.K., Chen, T., Amendola, M. \& van Steensel, B. Nucleic Acids Research 42, e168-e168 (2014).

37. Rueden, C.T. et al. BMC Bioinformatics 18, 529 (2017).

38. Schneider, C.A., Rasband, W.S. \& Eliceiri, K.W. Nat Methods 9, 671-675 (2012). 